\title{
Dynamique De La Végétation De Bamo Et Stocks De Carbone Dans La Mosaïque De Végétation
}

\author{
Adingra Odette Marie M. Anobla \\ Doctorante au Laboratoire de Botanique, UFR Biosciences ; \\ Université Félix Houphouët Boigny, Abidjan, Côte d'Ivoire \\ Justin Kassi N'Dja \\ Maître de Conférences, Laboratoire de Botanique, UFR Biosciences. \\ Université Félix Houphouët Boigny, Abidjan, Côte d'Ivoire
}

doi: 10.19044/esj.2016.v12n18p359 URL:http://dx.doi.org/10.19044/esj.2016.v12n18p359

\begin{abstract}
This study was conducted in Agboville, Ivory Coast. The objective was (1) to characterize the floristic composition, dynamics, and structure of tree diversity of postcultural fallows; and (2) establish the relationship between the diversity and storage of carbon in timber biomass. The study relied on a network of 50 temporary plots. There were 31 postcultural fallows and 19 plots of primary forest. This study has identified 417 plant species distributed in 306 genera and 83 families. Tree density within postcultural fallows varied between 1025 to 5975 stems / ha, and the analysis showed that the density increases with the age of the postcultural fallows. Tree sampling was non-destructive and to estimate $\mathrm{C}$ storage, an allomectric models for above and belowground biomasses was used. Mean estimate of carbon (C) stocks in biomass were $80.25 \mathrm{tC} /$ ha for groupe A (fallows from 4 to 8 years) and 256.5 tC / ha for group D (fallows from 14 to 24 years). These values remain far below those seen in the groups of primary forests with lateritic soil (1335.25 tC / ha). Results showed the ability of some postcultural fallows to store much C. It demonstrated that storage depends mainly on age and conserved species. This supports the idea of employing REDD+ processes in enhancing the ecological value associated with carbon.
\end{abstract}

Keyswords: Postcultural fallows, restoration, carbon stocks, Ivory Coast

\section{Resume}

La présente étude a été menée dans la région d'Agboville (Côte d'Ivoire). Elle a eu pour objectifs (1) de caractériser la composition floristique de la forêt ; (2) de reconstituer la dynamique de recolonisation des jachères post-culturales ; (3) de caractériser la structure de 
la diversité arborée; (4) d'évaluer la dynamique de stockage de carbone en fonction de l'âge des jachères. L'étude a portée sur 50 parcelles dont 31 relevés de jachères et 19 parcelles de forêts primaires. Un total de 417 espèces a été inventorié pour l'ensemble de la forêt classée. Celle-ci est repartie en 306 genres et 83 familles. La densité entre les jachères variée de 1025 (groupe A : 4-8 ans) à 5975 tiges/ha (groupe D : 1424 ans). Cette étude a permis de mieux connaître la dynamique et la structure de la forêt. Les estimations des stocks de $\mathrm{C}$ dans la biomasse ligneuse aérienne et souterraine à été faite à l'aide d'équation allométrique basé sur la méthode non destructive. Ils sont de 80,25 tC/ha pour le groupe de jachères de 4 à 8 ans et de 256,5 tC/ha pour le groupe de jachères âgées de 14 à 24 ans. Ces valeurs restent largement inférieures à celles observées dans les groupes de relevés de forêts primaires (forêts primaires sur sol ferrallitiques 1335,25 tC/ha). Ces données démontrent : (a) la capacité des jachères post-culturales à stocker du C (Carbone); (b) que ce stockage dépend principalement de l'âge, des essences conservées et des antécédents culturaux; et (c) que la valeur écologique liée au carbone pourrait être une opportunité de retombées financières en cas de paiement des services environnementaux procurés par ces types d'utilisation des terres dans le processus du REDD+.

Mots clés : Forêts secondaires, régénération, séquestration du carbone, Côte d'Ivoire

\section{Introduction}

Selon la FAO, en 2011, les forêts du monde couvraient environ 31\% des terres de la planète soit un peu plus de 4 milliards d'hectares, Ces forêts présentent une importance capitale d'autant plus qu'elles fournissent des biens et services qui sont essentiels pour 1,2 milliards de personnes dans le monde. Tayo (2014), souligne qu'elles constituent l'habitat naturel de la diversité biologique tant floristique que faunique, Mais cette richesse naturelle en Côte d'Ivoire est menacée par des activités qui augmentent avec la pression sur les forêts au point où celles-ci connaissent des taux de déforestation annuels de 1,86\% par an pendant la décennie 2000 (Koné et al., 2014). Les forêts ivoiriennes s'étendaient sur 16 millions d'hectares à la fin du $19^{\text {ème }}$ siècle. Elles sont évaluées à 2,7 millions d'hectares à la fin du $20^{\text {ème }}$ siècle (Koné et al., 2014).

La déforestation en Côte d'Ivoire a pour conséquence directe l'émission de $\mathrm{CO}_{2}$ dans l'atmosphère ce qui contribue significativement aux changements climatiques qui demeure jusqu'ici l'une des principales préoccupations de la communauté internationale. Selon la FAO (2008), l'agriculture et le changement d'affectation des terres, tel que le déboisement, représentent respectivement environ 13 et $17 \%$ du total des 
émissions des GES (gaz à effets de serres) résultant de l'activité humaine. Pour Tayo (2014), limiter la déforestation signifie non seulement moins de carbone $(\mathrm{C})$ relâché dans l'atmosphère, mais aussi l'habitat préservé pour de nombreuses espèces en voie d'extinction et des services écologiques essentiels maintenus. C'est pourquoi au cours de la dernière décennie, le besoin de réduire les effets néfastes du changement climatique à engendré un besoin d'information sur l'évolution dans le temps et dans l'espace de la couverture végétale. Dans l'optique de définir une politique climatique mondiale, des estimations fiables des stocks de carbone (C) dans les écosystèmes forestiers sont nécessaires (Torquebiau, 2002) et dans ce contexte, l'agroforesterie et la réhabilitation des jachères sont aujourd'hui perçues comme des options d'utilisation des terres pouvant contribuer à résoudre certaines menaces pesant sur l'environnement dans les pays tropicaux où la destruction des forêts est un enjeu majeur (Torquebiau, 2002). En matière d'environnement, ces systèmes offrent une gamme de services tels que la conservation de la biodiversité et des sols, le maintien de la fertilité des sols et la séquestration du C (Kassi, 2006 ; Tayo, 2014).

Face à ces enjeux économiques et écologiques, il devient essentiel de fournir de manière plus précise les estimations des stocks de $C$ forestier et les changements de ces stocks dans les forêts secondaires, et de comprendre le rôle de ces systèmes et notamment leur potentiel d'atténuation et d'adaptation au réchauffement climatique. Une valeur temporelle de stock de $\mathrm{C}$ « time averaged carbon stock » de chaque utilisation de terre devrait être évaluée et la valeur économique conséquente déduite sous forme de valeur actualisée nette (Minang et al., 2009). D’où l'intérêt de la présente étude qui contribuera à quantifier le C pouvant être stockée le long de notre chronoséquence.

L’objectif général de cette étude est de reconstituer la recolonisation post-culturale et de caractériser les stocks de C dans la mosaïque de végétation de la forêt.

Plus spécifiquement, il s'agira premièrement, de dresser la composition floristique et de reconstituer la dynamique de recolonisation post-culturale ;

-secondairement, de reconstituer la structure de la diversité arborée et d'établir les relations entre cette diversité et la capacité de stockage du carbone dans la biomasse ligneuse ;

- et enfin, évaluer la dynamique de stockage et les valeurs écologique et commerciale des jachères d’âges et les différents types de forêts primaires. 


\section{Méthodes d'études}

\section{Aire d'étude}

La forêt classée de Bamo ( $5^{\circ} 54^{\prime}$ et $5^{\circ} 55^{\prime} \mathrm{N}$ et $4^{\circ} 33^{\prime}$ et $\left.4^{\circ} 37 \mathrm{O}\right)$ est située à moins de trois $\mathrm{km}$ de la ville d'Agboville. Il s'agit d'une forêt dense semi-décidue soumise à un climat tropical sub-humide, appartenant au secteur mésophile d'après les subdivisions établies par Guillaumet \& Adjanohoun (1971). Elle couvre 255 hectares sur un socle schisto-birrimiengranitique sur lequel se sont développés des sols ferrallitiques et hydromorphes. Le climat (moyennes annuelles : $26,72^{\circ} \mathrm{C}$ et $1585,35 \mathrm{~mm}$ ), détermine une végétation potentielle de forêt dense humide semi-décidue.

\section{Méthode}

Le plan d'échantillonnage a été conçu de manière à inventorier des forêts primaires ( $n=19$ relevés) et des forêts secondaires ou jachères de différents âges ( $\mathrm{n}=31$ relevés). Dans la forêt classée de Bamo, nous n’avons pas observé de jachère de moins de 4 ans. La taille des échantillons était de $50 \mathrm{~m}$ x $30 \mathrm{~m}\left(1500 \mathrm{~m}^{2}\right)$. Les relevés de végétation consistaient en une liste exhaustive de toutes les espèces vasculaires présentes dans la surfaceéchantillon selon les principes de la phytosociologie synusiale (Gillet et al., 1991). Des inventaires itinérants complémentaires ont été menés dans toutes les parties de la forêt y compris les cultures. Ces inventaires ont permis de compléter la liste floristique. Toutes les espèces ont été identifiées. La nomenclature adoptée est celle APG III (2009). Les relevés phytosociologiques sont couplés d'un inventaire forestier. Nous avons effectué des mesures dendrométriques concernant la hauteur et la circonférence à $1,30 \mathrm{~m}$ du sol à l'aide d'un mètre ruban. Seuls les ligneux dont le diamètre est supérieure à $05 \mathrm{~cm}$ sont pris en compte. La distribution des différents individus par classe de hauteur $\left(\mathrm{H}_{1} \leq 5 \mathrm{~m}\right.$, de $5 \mathrm{~m}$ à $15 \mathrm{~m}=\mathrm{H}_{2}$, de 15 à $25 \mathrm{~m}=\mathrm{H}_{3}$ et $\left.\mathrm{H}_{4}>25 \mathrm{~m}\right)$ ainsi que par classe de diamètre $(5 \mathrm{~cm}, 10$ $\mathrm{cm}, 15 \mathrm{~cm}$ et $30 \mathrm{~cm}$ et plus) a été effectuée dans les parcelles $(20 \mathrm{~m}$ x $20 \mathrm{~m}=$ $400 \mathrm{~m}^{2}$ ) qui ont servies à l'estimation du stock de carbone dans la biomasse totale des ligneux sur pied. Nous prenons les moyennes des hauteurs et des diamètres pour le calcul du stock de carbone.

\section{Analyses des données}

Nous avons établi un catalogue floristique reprenant toutes les espèces présentes dans les 50 relevés de végétations, auxquelles nous avons adjoint les espèces supplémentaires répertoriées au cours des prospections itinérantes. En vue d'une analyse exploratoire multidimensionnelle des relevés phytosociologiques, nous avons retenu comme variables explicatives les facteurs suivants : âge de la jachère, type de sol, densité de la forêt primaire. Les relevés ont d'abord fait l'objet d'une classification hiérarchique 
suivant l'indice de dissimilarité de Bray-Curtis de manière à analyser l'assemblage des espèces en communautés végétales, suivie d'une analyse des espèces indicatrices (Dufrêne \& Legendre, 1997) pour caractériser ces dernières sur le plan floristique. Les groupes hiérarchiques et les axes des plans factoriels obtenus ont été interprétés au vu des données environnementales, grâce à des tests de Kruskal-Wallis et de corrélation de Spearman respectivement $(\mathrm{p}<0,05)$.

Nous avons par la suite calculé, la densité et la surface terrière. Les quantités de biomasse aérienne des différents individus ont été déterminées à l'aide du modèle standard de régression de Chave et al. (2014) pour les forêts tropicales humides selon la formule suivante : AGB $(\mathrm{Kg})=0,0559 \times \mathrm{\rho D}^{2} \mathrm{H}$. La biomasse totale (TB) des ligneux sur pied sera alors estimée en sommant les deux valeurs de biomasse aérienne et souterraine selon la formule suivante : $\mathrm{TB}=\mathrm{ABG}+\mathrm{BGB}$. La biomasse totale estimée à partir des différentes équations a été convertie en stock de carbone séquestré correspondant en la multipliant par 0,47 (GIEC, 2006).Les valeurs de stocks de carbone trouvées à l'échelle des différentes aires d'échantillonnage ont été extrapolées à l'hectare $\left(10000 \mathrm{~m}^{2}\right)$.Les valeurs écologiques « carbone » liée aux forêts secondaires et aux forêts primaires. La tonne équivalente de $\mathrm{CO}_{2}$ séquestrée sur le marché des MDP est de 6\$ (Glenn, 2008). Toutes les analyses ont été réalisées grâce au logiciel $R^{\circledR}$.

\section{Résultats}

\section{Analyse floristique}

Les relevés phytosociologiques suivis de l'inventaire forestier ont permis de recenser 417 espèces végétales pour l'ensemble de la forêt classée, réparties en 306 genres et 83 familles.

\section{Dynamique des jachères post-culturales}

Les jachères post-culturales ou écosystèmes culturaux " abandonnés » subissent une dynamique progressive de la végétation. Le processus de recolonisation de la jachère s'effectue suivant plusieurs étapes successives. La classification hiérarchique ascendante des relevés (Figure 1) obtenue suivant l'indice de dissimilarité de Bray-Curtis confirme les différents types de jachères identifiés sur le terrain. Au seuil de signification d'environ75 \%, l'analyse permet de dégager sept (7) grands groupes de jachères. Le groupe $\mathrm{A}$ est constitué des jachères de 4 à 8 ans. Le groupe $B$ est celui des jachères de 9 à 13 ans. Les jachères de 14 à 20 ans forment le groupe $C$ alors que le groupe $\mathrm{D}$ est constitué de jachères presque matures (plus de 14 ans, c'est-à-dire les jachères de 15 à 24 ans). Ces groupes coïncident globalement avec les quatre (4) classes d'âge des jachères (de A à D) et les trois (3) types forestiers ( $E$ : forêt primaire sur sol ferralitique, $F$ : Forêt galerie et $G$ : forêt primaire sur sol 
hydromorphe). L'âge de la jachère est le principal déterminant des groupes $(\mathrm{H}=41,8 ; \mathrm{p}<0,001)$, avec une séparation nette entre les jeunes jachères (groupes $\mathrm{A}$ à $\mathrm{D}$ ) des autres relevés (relevés de forêts primaires plus quatre relevés de jachères âgées : 25-27 ans). Les groupes $A$ et $B$ regroupent les plus jeunes stades de la succession. Ils sont situés dans la première branche du cluster. Ils sont situés dans la première branche du cluster. Ces jeunes stades, sont peu structurés, caractérisés par des espèces de la strate sous-arbustive dont la taille n'excède pas cinq mètres. Ces jeunes jachères sont largement dominées par une espèce invasive, Chromolaena odorata, qui occupe à elle seule, plus de $85 \%$ de la couverture végétale au niveau du groupe I.

Le quatrième groupe ( $\mathrm{D}$ : jachères de 14 - 24 ans) est clairement séparé des trois autres groupes de jeunes jachères. Il est d'ailleurs situé dans la deuxième branche du cluster. Ce groupe est plus riche en espèces que les trois premiers groupes (groupes $\mathrm{A}, \mathrm{B}$ et $\mathrm{C}$ ). La CAH sépare très nettement les relevés de forêt primaire (d'une façon générale) en fonction du type de sol (ferrallitique vs hydromorphe vs galerie ou bas-fonds) puisque chacun des trois groupes semble avoir une spécificité au niveau de la composition floristique. Ce qui n'est pas très forcement le cas au niveau des jachères. Il en est de même pour les forêts primaires sur sol ferralitique (E) et les trois relevés de jachères de $25-27$ ans ( $\left.E^{\prime}\right)$. 
Albiziaadianthifolia S1,

S2

Ficus exasperata $\mathrm{S} 1$

$C: 14-20$

Ceibapentandra A1, A2 ans

Albiziaadianthifolia A2, S1,

${ }_{\text {Albiziaferruginea } \mathrm{S} 1}^{\mathrm{B}}{ }^{\mathrm{S} 2}$ 9-13

Albiziazygia S2, S1

Albiziaadianthifolia

S1

Albiziaferruginea S1

Albiziazygia S2, S2

Albiziaadianthifolia A1,

A2

Ficus exasperata $S 1$

Albiziaferruginea S1

Albiziazygia $\mathrm{S} 2$

A : 4-8

ans

$\mathrm{P} 23$

$\mathrm{P} 46$

P32

P44

P16

P37

P19

P29

P14

P17

P18

P42

P9

P31

P15

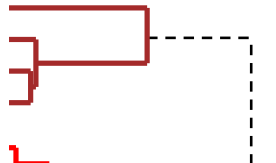

$15-24$

P24

P11

P12

P3

P39

ans

P36

$\mathrm{P} 40$

Albiziaadianthifolia

A2

Ceibapentandra A1,

Ceibapentandra A1, A2

Celtiszenkeri A1 E : Forêt

Miliciaregia A1, A2, S1, SZer

Terminaliaivorensis A1, A2,

\section{Ceibapentandra A1 \\ Celtiszenkeri A1}

Terminaliaivorensis

A1

Ceibapentandra A1

Treculiaafricana A1

Terminaliaivorensis

A1

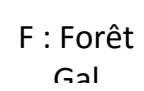

$\mathrm{P} 50$

E' : 25-27 P48

ans

P8

P28

P6

P41

P21

P4

P10

P45

P27

$\mathrm{P} 43$
$\mathrm{P} 13$

P30

P34

G : Forêt P47

Hyd

\section{P47
P38}

$\mathrm{P} 7$
$\mathrm{P} 7 \mathrm{n}$
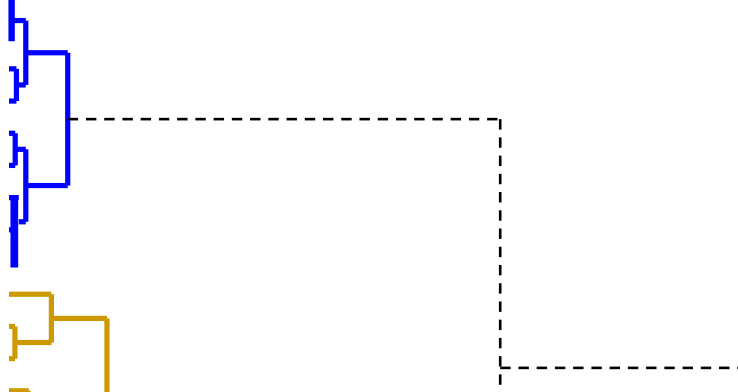

$1--$
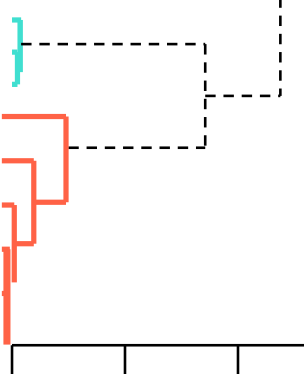

$\begin{array}{lllllll}0 . & 0 . & 1 . & 1 . & 2 . & 2 . & 3 . \\ 0 & 5 & 0 & 5 & 0 & 5 & 0\end{array}$

Figure 1 : Dendrogramme de la classification hiérarchique ascendante des 50 relevés avec les principales espèces pour l'analyse structurale

Légende : Fer : Ferralittique ; Gal : Galerie ; Hyd : Hydromorphe; A1: arbres de $25 \mathrm{~cm}$ :; A2: arbres de 15 - $25 \mathrm{~cm}$; S1: petits arbres de $5-15 \mathrm{~cm}$; S2: arbuste et petit arbre de $5 \mathrm{~cm} \leq$ 


\section{Structure diamétrique des arbres et arbustes dans les relevés}

La densité des espèces de $\mathrm{dbh} \geq 5 \mathrm{~cm}$, recensées dans les parcelles et classées en fonction des groupes de relevés issues de la $\mathrm{CH}$ ou CAH est indiquée dans la figure 2. Dans cette partie des travaux, nous avons pris en compte les arbres rémanents $(\leq 0 v s$. $\geq 3)$. Les arbres rémanents sont des arbres épargnés lors de la création des champs. Ces arbres ont une circonférence $\geq 30 \mathrm{~cm}$ dans les jeunes jachères. Dans les jeunes jachères des groupes $\mathrm{A}$ et $\mathrm{B}$, les espèces qui ont une circonférence $\geq 5 \mathrm{~cm}$ sont généralement peu importantes. Il s'agit des espèces comme Albizia spp., Ficus exasperata, Musanga cecropioide, Trema orientalis. La densité des espèces de dbh $\geq 5 \mathrm{~cm}$ passe de 1025 tiges / ha dans les jeunes jachères de 4 à 8 ans à 5975 tiges/ ha dans les jachères de 14 à 24 ans. Dans les trois jachères de 25-27 ans, la densité est de 4175 tiges/ ha. Ces différentes valeurs sont largement inferieures à celles observées dans les différents relevés de forêts (forêts galeries 6625 tiges/ ha et forêts primaires sur sol ferralittiques : 19075 tiges/ ha). Nous n'avons par pris en compte les relevés de forêt sur les sols hydromorphes. Car, en observant la CAH (Figure 1), nous voyons que les relevés des jachères de 25- 27 ans sont plus proche des relevés de forêts sur sol ferralitiques

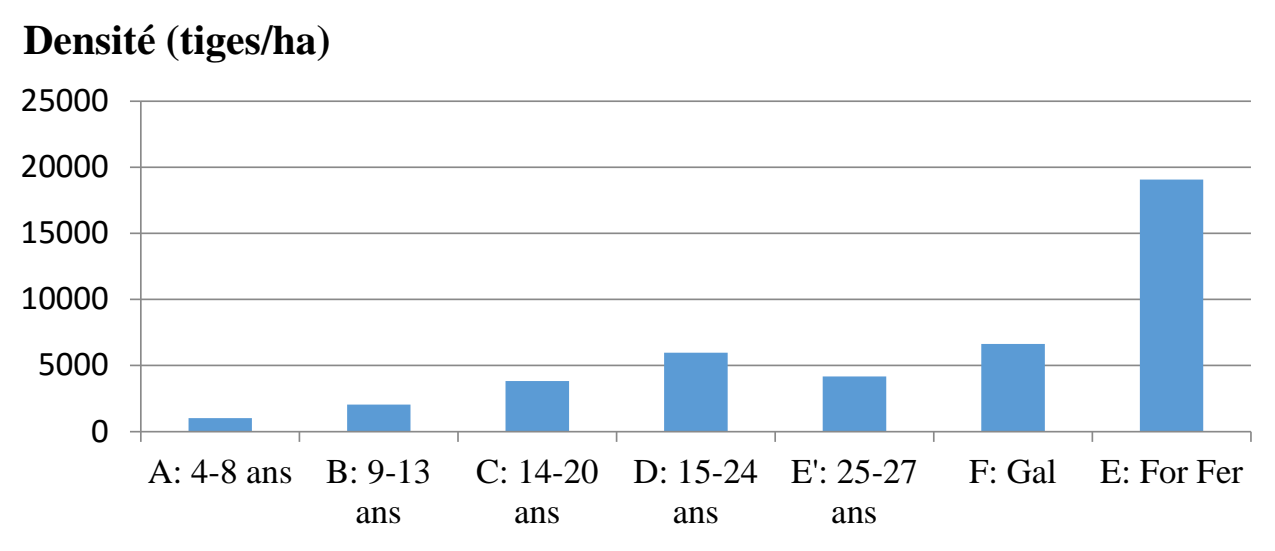

Figure 2 : Densité des espèces inventoriées en fonction des groupes issus de la CAH

que les forêts sur sol hydromphes. Le test de corrélation de Spearman $(\mathrm{P}<0,05)$ présenté dans le tableau $\mathrm{I}$, indique les paramètres environnementaux qui influencent significativement la composition floristique et la structure (exemple, le recouvrement des strates de végétation) des jachères, indépendamment de l'effet de l'âge. Ce qui n'est pas le cas du type de culture, de la taille du champ, du type de sol et dans une moindre mesure la durée de la dernière culture dans notre contexte de petits champs exploités clandestinement. 
Tableau I: Corrélation entre la richesse spécifique, le recouvrement et les différentes variables. Légende : ns : non significatif ; ** : $\mathrm{P}<0,05 ; * * *: \mathrm{P}<0,01$

\begin{tabular}{|c|c|c|c|c|c|}
\hline & $\begin{array}{l}\text { Richesse } \\
\text { spécifique }\end{array}$ & $\begin{array}{c}\text { Recouvrement } \\
\mathrm{S}_{1}\end{array}$ & $\begin{array}{c}\text { Recouvrement } \\
\mathrm{S}_{2}\end{array}$ & $\begin{array}{c}\text { Recouvrement } \\
\mathrm{A}_{2}\end{array}$ & $\begin{array}{c}\text { Recouvrement } \\
\mathrm{A}_{1}\end{array}$ \\
\hline $\begin{array}{l}\text { Type de } \\
\text { culture }\end{array}$ & $\begin{array}{c}0,359 \\
\text { ns }\end{array}$ & $\begin{array}{c}0,275 \\
\text { ns }\end{array}$ & $\begin{array}{c}0,551 \\
\text { ns }\end{array}$ & $\begin{array}{c}0,243 \\
\text { ns }\end{array}$ & $\begin{array}{c}0,174 \\
\text { ns }\end{array}$ \\
\hline $\begin{array}{l}\text { Type de } \\
\text { sol }\end{array}$ & $\begin{array}{c}0,875 \\
n s\end{array}$ & $\begin{array}{c}0,995 \\
\text { ns }\end{array}$ & $\begin{array}{c}0,192 \\
\text { ns }\end{array}$ & $\begin{array}{c}0,935 \\
\text { ns }\end{array}$ & $\begin{array}{c}0,999 \\
\text { ns }\end{array}$ \\
\hline $\begin{array}{l}\text { Taille du } \\
\text { champ }\end{array}$ & $\begin{array}{c}0,610 \\
n s\end{array}$ & $\begin{array}{c}0,962 \\
n s\end{array}$ & $\begin{array}{c}0,565 \\
\text { ns }\end{array}$ & $\begin{array}{c}0,492 \\
n s\end{array}$ & $\begin{array}{c}0,176 \\
n s\end{array}$ \\
\hline Age & $\begin{array}{c}1,028 \\
* * *\end{array}$ & $\begin{array}{c}1,922 \\
* * *\end{array}$ & $\begin{array}{c}7,949 \\
* * *\end{array}$ & $\begin{array}{c}1,077 \\
* * *\end{array}$ & $\begin{array}{c}1,275 \\
* * *\end{array}$ \\
\hline $\begin{array}{l}\text { Durée de } \\
\text { la dernière } \\
\text { culture }\end{array}$ & $\begin{array}{c}0,078 \\
\text { ns }\end{array}$ & $\begin{array}{c}0,032 \\
* *\end{array}$ & $\begin{array}{c}0,0501 \\
\mathrm{~ns}\end{array}$ & $\begin{array}{c}0,13 \\
n s\end{array}$ & $\begin{array}{l}0,198 \\
\text { Ns }\end{array}$ \\
\hline $\begin{array}{c}\text { Densité } \\
\text { forestière }\end{array}$ & $\begin{array}{c}0,023 \\
* * *\end{array}$ & $\begin{array}{c}0,110 \\
* *\end{array}$ & $\begin{array}{c}0,304 \\
* *\end{array}$ & $\begin{array}{c}0,160 \\
* *\end{array}$ & $\begin{array}{c}0,064 \\
* *\end{array}$ \\
\hline $\begin{array}{l}\text { Nombre de } \\
\text { rémanents }\end{array}$ & $\begin{array}{c}0,002 \\
* * *\end{array}$ & $\begin{array}{c}0,013 \\
* *\end{array}$ & $\begin{array}{c}0,058 \\
* *\end{array}$ & $\begin{array}{c}0,017 \\
* *\end{array}$ & $\begin{array}{c}0,006 \\
* * *\end{array}$ \\
\hline
\end{tabular}

qui concerne la distribution des arbres et arbustes par classe de diamètre le long de la chronoséquence en fonction de l'âge (Figure 3). Les jeunes jachères sont caractérisées par des individus de petits diamètres d'où la structure de $\mathrm{J}$ renversée indiquant une population jeune en pleine reconstitution après l'abandon des champs et ceci pendant tous les stades d'évolution des jachères. Seules les parcelles d'âge supérieur à 9 ans possèdent les 4 classes de diamètres. Les rémanents qui sont maintenus lors de la création des champs font que dans les jachères de 4- 8 ans nous avons les troncs d'arbres de plus de $30 \mathrm{~cm}$. Au cours de la régénération, les plus grosses tiges s'observent plus dans les vieilles jachères. Il s'agit généralement des espèces à croissance rapide comme : Albizia adianthifolia, Albizia zygia, Ficus exasperata, Harungana madagascariensis, Musanga cecropioide, Trichilia monadelpha... La distribution des arbres et arbustes au niveau des relevés de forêt, montre que ces forêts disposent de plusieurs tiges d'avenir pour assurer leur régénération. C'est la principale caractéristique d'un peuplement forestier supposé être en équilibre, avec beaucoup de tige de petits diamètres et peu de tiges de gros diamètre. La stratification du couvert (non représentée) est le même que celui de la distribution diamétrique puis que nous avons utilisé les moyennes des hauteurs et des diamètres. 


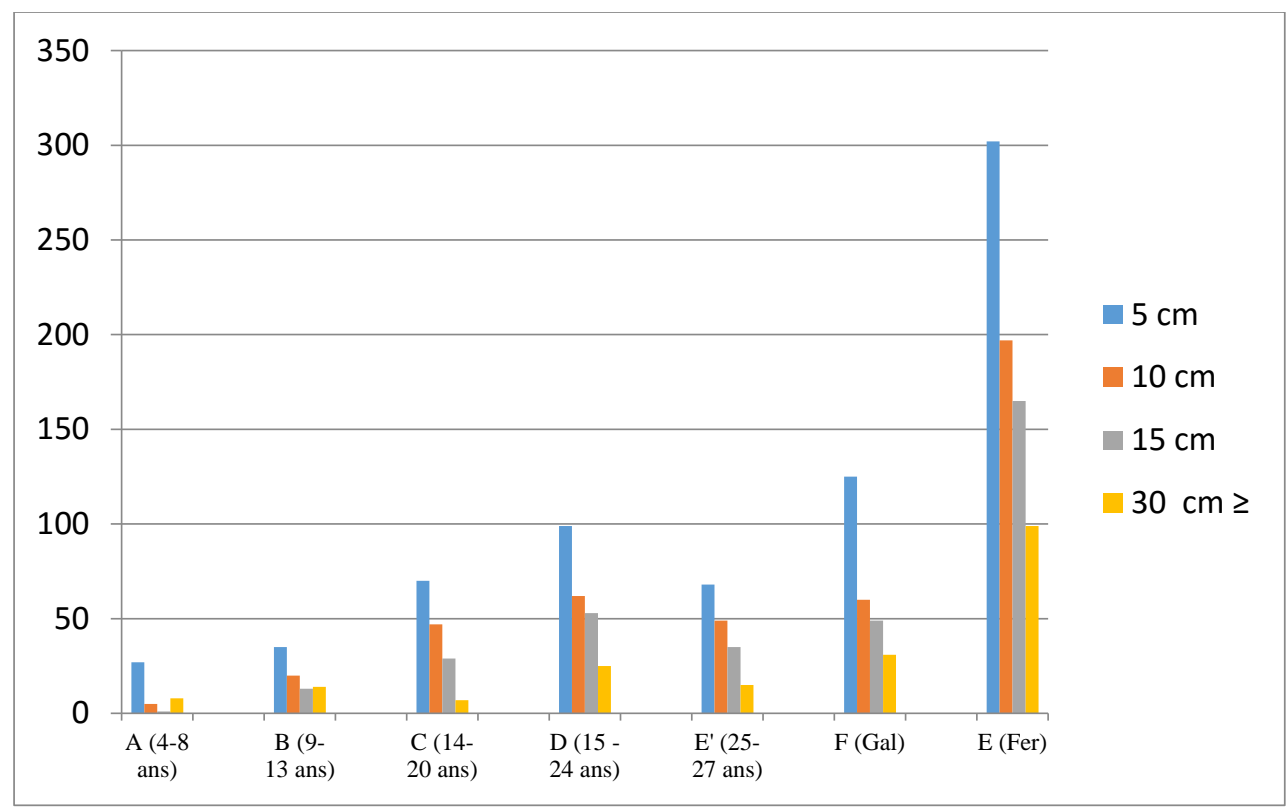

Figure 3 : Distribution diamètrique des arbres et arbustes dans les relevés Légende : Gal : Forêt galerie ; Fer : Forêt sur sol ferrallitique

\section{Relation entre diversité et capacité de stockage du carbone Surface terrière}

Les valeurs des surfaces terrières suivent la même tendance observée pour les densités. Ainsi, les surfaces terrières varient de 16,8775 à 301,195 $\mathrm{m}^{2}$ /ha le long de la chronoséquence (Tableau II).

\section{Estimation du Carbone séquestré dans les différents groupes de végétation}

Le tableau II présente les paramètres structuraux, la biomasse aérienne et souterraine et le $\mathrm{CO}_{2}$ dans les différents groupes de relevés issus de la $\mathrm{CAH}$. Nous pouvons noter que les estimations des stocks de $\mathrm{C}$ dans les jachères du groupe $\mathrm{A}$ aux groupes $\mathrm{D}$ et $\mathrm{E}$ ' sont significativement différents. Ainsi, le carbone séquestré dans les jachères passe de $80,25 \mathrm{tC} / \mathrm{ha}$ dans groupe $\mathrm{A}$ à $256,5 \mathrm{tC} / \mathrm{ha}$ dans les jachères du groupe $\mathrm{D}$ et $143 \mathrm{tC} / \mathrm{ha}$ dans les jachères du groupe E'. Ces valeurs restent largement inférieures aux quantités de carbone séquestré dans les deux groupes de forêts (forêt galerie : 449,5 tC/ha et forêt sur sol ferralitique : 1335,25 tC/ha). Les variations des valeurs totales d'équivalent $\mathrm{CO}_{2}$ suivent les mêmes tendances observées pour les stocks de carbone, dans les différents groupes de relevés (Tableau II). Nous observons une augmentation des stocks avec l'ancienneté des jachères. Les valeurs les plus grandes au niveau des jachères qui sont de 774,75; 938,5 et 523,5 $\mathrm{tCO}_{2} /$ ha sont obtenues dans les jachères de 14 ans et plus regroupés respectivement dans les groupes $\mathrm{C}$, D et $\mathrm{E}$ ' issus de la CAH. 


\section{Valeur écologique liée au carbone des jachères}

Les stocks moyens de $C$ séquestrés en fonction de notre chronoséquence ont été convertis en valeur écologique telle que le préconise le marché de carbone dans la REDD+ pour obtenir les Certificat d'Emissions Réduites (CER) qui seront traduits en tonnes équivalentes de $\mathrm{CO}_{2}$ qui est la monnaie d'échange sur le marché du $\mathrm{C}$. Par application d'une valeur de 6 \$ par unité de tonne équivalente de $\mathrm{CO}_{2}$ séquestrée (Glenn, 2008).

La figure 4 présente la valeur du service écologique de séquestration du $\mathrm{C}$ en cas de paiement dans les différentes jachères post-culturales. Les stocks de $\mathrm{CO}_{2}$ dans les jachères variant de 293,25 à 938,5 tCO2/ha. La plus grande valeur écologique à été trouvé dans les forêts secondaires du groupe D (5631 \$/ha). Cette valeur écologique étant la valeur du C s’il est vendu sur le marché, elle croît avec l'âge des jachères donc progressivement des milieux récemment dégradés vers les milieux les moins dégradés avec les stocks les plus élevés.

Tableau II: Paramètres structuraux, biomasse aérienne, biomasse souterraine et masse de $\mathrm{CO}_{2}$ dans les différents groupes de relevés

\begin{tabular}{|c|c|c|c|c|c|c|c|}
\hline & \multicolumn{7}{|c|}{ Groupes de relevés issus de la CAH et de la DCA } \\
\hline & A (4-8 ans) & $\begin{array}{c}\mathrm{B}(9- \\
13 \text { ans) }\end{array}$ & $\begin{array}{c}\mathrm{C}(14-20 \\
\text { ans) }\end{array}$ & $\begin{array}{c}\mathrm{D}(15-24 \\
\text { ans) }\end{array}$ & $\begin{array}{l}E^{\prime}(25-27 \\
\text { ans) }\end{array}$ & F (Gal) & E (Fer) \\
\hline $\begin{array}{l}\text { Nombre } \\
\text { de } \\
\text { relevés }\end{array}$ & 7 & 7 & 4 & 8 & 3 & 3 & 10 \\
\hline $\begin{array}{l}\text { Densité totale } \\
\text { des arbres } \\
\text { (tiges) }\end{array}$ & 41 & 82 & 153 & 239 & 167 & 265 & 763 \\
\hline $\begin{array}{l}\text { Surface terrière } \\
\text { totale }\left(\mathrm{m}^{2} / \mathrm{ha}\right)\end{array}$ & 16,8775 & 36,11 & 37,8275 & 84,585 & 54,9 & 94,2975 & 301,195 \\
\hline $\begin{array}{c}\text { Biomasse } \\
\text { aérienne (Kg) }\end{array}$ & 124463,75 & 142819 & 329337,5 & 398294 & 222260 & 698437 & 2074973,25 \\
\hline $\begin{array}{c}\text { Biomasse } \\
\text { souterraine (Kg) }\end{array}$ & 46051,5 & 52843 & 121854,75 & 147368,75 & 82236,25 & 258421,75 & 767740 \\
\hline $\begin{array}{l}\text { Biomasse totale } \\
(\mathrm{Kg})\end{array}$ & 170515,25 & 195662 & 451192,25 & 545662,75 & 304496,25 & 956858,75 & 2842713,25 \\
\hline $\begin{array}{l}\text { Carbone } \\
\text { sequester } \\
(\mathrm{tC} / \mathrm{ha})\end{array}$ & 80,25 & 91,75 & 211,75 & 256,5 & 143 & 449,5 & 1335,25 \\
\hline $\begin{array}{l}\mathrm{Co}_{2} \text { équivalent } \\
\text { (tCO } \mathrm{tCO}_{2} / \mathrm{ha} \text { ) }\end{array}$ & 293,25 & 335,75 & 774,75 & 938,5 & 523,5 & 1645,5 & 4890,25 \\
\hline
\end{tabular}




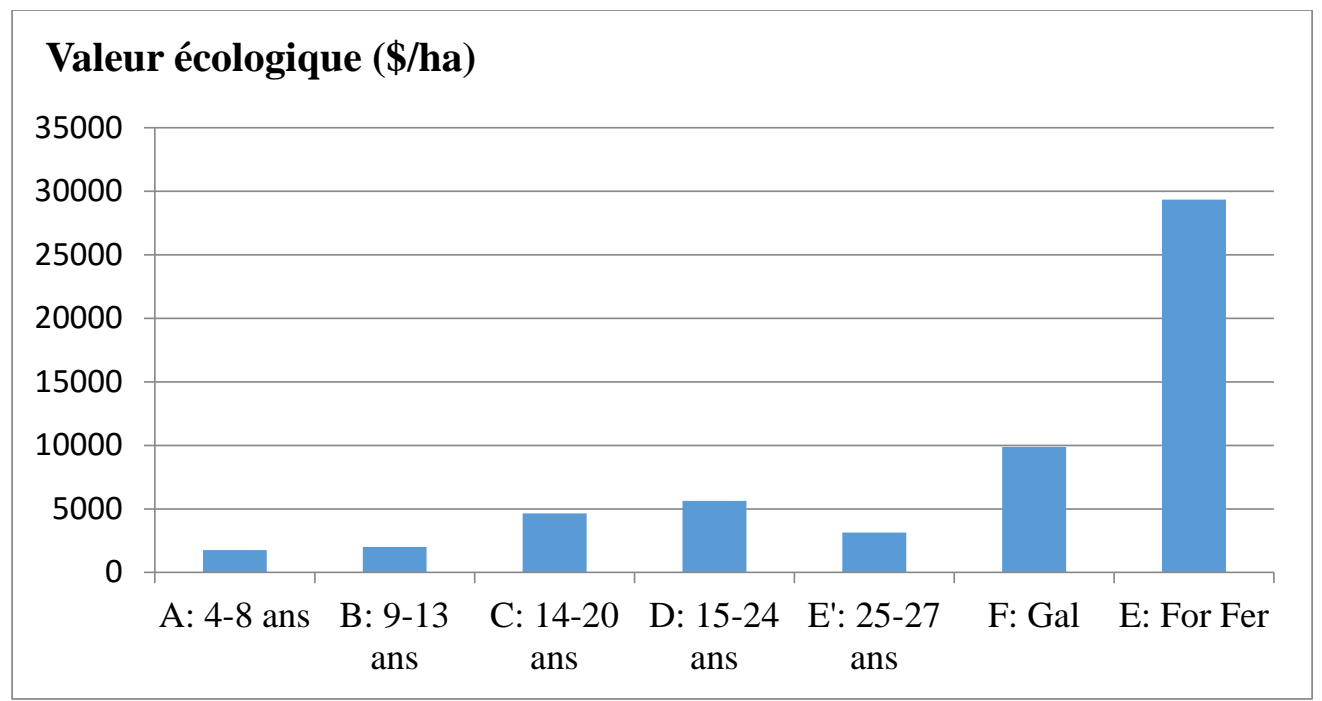

Figure 4: Evaluation de la valeur écologique des jachères post-culturales

\section{Discussion}

En Côte d'Ivoire, pendant longtemps, la dégradation du milieu naturel n'a pas vraiment préoccupé la population et la destruction de la végétation était considérée comme un bien pour l'activité agricole, indispensable au développement économique (Aké-Assi, 1998). Dans une optique de développement durable nous avons réalisé ces travaux pour voir quel peut-être l'apport des jachères post-culturales dans l'économie et dans la conservation de la biodiversité devant la menace de raréfaction, voire de disparition, de nombre d'espèces et d'écosystèmes naturels. Au plan floristique, cette étude a évalué la diversité floristique de la forêt classée et a fourni la première liste complète de sa flore vasculaire: 417 espèces réparties dans 83 familles et 307 genres. Parmi celles-ci, 39 (soit 9,35 \%) sont endémiques Ouest africaines. Selon Boukpessi (2013), le temps de repos qui suit la culture se caractérise par une dynamique progressive de la végétation. $\mathrm{Au}$ niveau de la régénération, nos résultats montrent que de la jachère à Chromolaena odorata de 4 à 8 ans, nous sommes passé progressivement à une jeune forêt secondaire (C : 14-20 ans) et enfin à une forêt secondaire mature ( $E^{\prime}: 25-27$ ans) qui met en place la structure forestière qui prendra les caractéristiques floristiques de la forêt primaire dont elle est issue. Des études entreprises en Côte d'Ivoire ont abouti aux résultats similaires (Kahn, 1982). Ainsi, les jachères post-culturales, lorsqu'elles sont bien conservées peuvent avoir de nombreuses fonctions socioéconomiques et environnementales très importantes à l'échelle mondiale, nationale et locale. Ainsi, plusieurs études ont démontré que les jachères post-culturales séquestrent du C notamment celle de (Saïdou, 2012) de même que les systèmes agroforestiers séquestrent également du C notamment celle de 
Tayo (2014) et peuvent offrir des opportunités d'atténuation au changement climatique en synergie avec l'adaptation. Nos travaux montrent que les stocks de carbone le long de la chronoséquence varie de 80, 25 tC /ha (jachères de 4-8 ans) à 256,5 tC /ha pour les jachères du groupe D (14 - 24 ans). Au niveau des forêts, nos travaux montrent que le stock de carbone dans le groupe $\mathrm{E}$ est de 1335,25 tC /ha. Le long de la chronoséquence, l'équivalent $\mathrm{CO}_{2}$ stocké dans les jeunes jachères du groupe $\mathrm{A}$ (4-8 ans) est 293,25 $\mathrm{tCO}_{2}$ /ha et de 938,5 $\mathrm{tCO}_{2}$ /ha pour les jachères du groupe $\mathrm{D}$ (15-24 ans), ce qui témoigne du rôle compensatoire des espèces arborescentes, dans les émissions du dioxyde de carbone issu des activités anthropiques que peuvent jouer les jachères post-culturales. Au niveau des forêts primaires, l'équivalent $\mathrm{CO}_{2}$ stocké est de 4890,25 tCO 2 /ha. Le stock de carbone dans les forêts galeries est 449,5 tC /ha pour trois parcelles contre 1335,25 tC /ha. Il en est de même pour l'équivalent de $\mathrm{Co}_{2}$ stocké dans ces deux groupes de forêts. Cette différence observée entre les deux types de forêts est probablement due aux conditions climatiques plus humides dans les forêts galeries. Mais des études ultérieures seront nécessaires pour donner une explication beaucoup plus précise. Selon Nolte et al. (2001), la capacité de stockage du carbone contenu dans une forêt primaire est de 275 tC /ha. Il est de 208tC /ha dans les forêts secondaires presque « reconstituées ». Dans les forêts secondaires âgées, il est de 151 tC /ha. Dans les forêts secondaires jeunes, il est de $108 \mathrm{tC} / \mathrm{ha}$. Le stock de carbone séquestré calculé dans le cadre de la présente étude est inférieur à ceux de Nolte et al. (2001). Car dans les jeunes jachères par exemple, nous avons 80,25 tC /ha pour 7 relevés soit 26,75 tC /ha par relevé contre 108 tC /ha dans les travaux de Nolte et al. (2001). Dans les 3 jachères nous avons obtenus dans nos travaux 143 tC /ha soit 46,67 tC /ha par relevé contre $151 \mathrm{tC} /$ ha dans les travaux de Nolte et al. (2001). Au niveau des forêts primaires, nous avons 1335,25 tC /ha pour les dix relevés soit 133, 52 tC /ha par relevé contre 275 tC /ha dans les travaux de Nolte et al. (2001). Cet écart s’explique principalement par la méthode utilisée pour les mesures de diamètre et de hauteur dans nos travaux. Au niveau des hauteurs et diamètres, nous avons établi des classes de diamètres et de hauteurs d'une part, et d'autre part, toutes les hauteurs supérieures à 30 m ont été ramenées à $30 \mathrm{~m}$. Il est de même au niveau des classes de diamètres. Tous les diamètres supérieurs à $30 \mathrm{~cm}$ ont été ramenés à $30 \mathrm{~cm}$. Nous n'avons pris en compte que les individus de $5 \mathrm{~cm}$ au niveau du diamètre et de 5 mètres au niveau de la hauteur. Cela a aussi, contribué à une baisse de la densité et qui a agit sans aucun doute sur le résultat final. Secondairement, on peut s'interroger sur l'originalité de nos forêts primaires. Car, au moment du classement de la forêt en 1929, il existait des champs. La forêt a aussi connue des enrichissements en espèces comme (Aucoumea klaineana pendant les premières années de son classement. 
Les valeurs écologiques trouvées dans les jachères sont très encourageantes. Il s'avère nécessaire que les services en charge du développement rural sensibilisent les populations pour une meilleure gestion de ces post-culturales dans la perspective de la réduction des gaz à effet de serre. Nous sommes d'accord avec Saïdou (2012) pour dire que, le développement des pratiques culturales préservant les arbres est à promouvoir pour une amélioration de la productivité des cultures associées et plus tard des jachères lorsque celle-ci seront laissées au repos. De nombreux travaux existent sur la séquestration de carbones dans les agrosystèmes en Afrique. Il s'agit entre autres des travaux dans les parcs à karité du NordCameroun (Peltier et al., 2007) et au Mali Traoré et al. (2004).

\section{Conclusion}

La succession secondaire post-culturale a été étudiée le long d'une chronoséquence de 31 jachères incluses dans une matrice de forêt dense semi-décidue. Les champs abandonnés en pleines forêt, ne présentent pas de difficultés pour reconstituer une forêt proche de celles dont elles sont issues. L'étude permet également de faire une estimation des stocks de carbone dans les diverses composantes des jachères post-culturales. Les principaux résultats montrent que ces jachères d'âges variées emmagasinent une quantité importante de carbone soit 80,25 tC /ha pour les jeunes jachères et 256,5 tC /ha pour les jachères âgées se trouve accumulé dans la biomasse aérienne et dans la biomasse souterraine. Ces résultats confirment l'énorme potentiel des jachères post-culturales dans la séquestration du carbone. Le développement des pratiques culturales préservant les arbres est à promouvoir pour une amélioration de la productivité des cultures associées et plus tard des jachères lorsque celle-ci seront laissées au repos. Cependant, le potentiel à contribuer aux stocks de carbone des jachères post-culturales d'une part, et d'autre part, des espèces africaines, est encore peu connu.

\section{References:}

Aké-Assi L. Espèces rares et en voie d'extinction de la flore de la Côte d’Ivoire. Monogr. Syst. Bot. Missouri Botanic Garden 25: 461-463, 1998.

APG III. An update of the Angiosperm Phylogeny Group classification for the

orders and families of flowering plants. Botanical Journal of the Linnean Society 161: 105-121, 2009.

Boukpessi T. Diversité floristique, structure et dynamique des agrosystèmes " abandonnes » de la Préfecture de Sotouboua (Centre-Togo). Rev. CAMES 1 : 176-197, 2013.

Chave J., Rejou- Mêchain M., Burquez A., Chidumayo E., Colgan M., Delitti B.C. W., Duque A., EiD T., Fearnside P., Fears L.M., Goodman C. 
R., Henry M., Inez-Yrizar A., Mugasha W., Mullerlandau C. H., Mencuccini M. Nelson WB., Ngomanda A., Noguiera E., Malvavaissi O.E., Pélissier R., Ploton P., Ryan C., Saldarriga J. \& Vieilledenti G. Improved allometric models to estimate the aboveground biomass of tropical trees. Global Change Biology 20: 3177-3190, 2014.

Dufrêne M. and Legendre P. Species assemblages and indicator species: the need for a flexible asymetrical approach. Ecological Monographs 67: 345366, 1997.

FAO. Financial Mechanisms for Adaptation to and Mitigation of Climate Change in the Food and Agriculture Sectors. Paper presented at the High Level Conference on World Food Security: The Challenges of Climate Change and Bioenergy, Rome, 3-5 June. 28 p, 2008.

FAO. State of the world's forests. FAO (Rome, Italy), 164 p, 2011.

GIEC. Guide pour l'inventaire national des gaz à effet de serre ; agriculture, foresterie et autre usage des terres. Institute for Global Environnemental Strategies, Japon 4: 46-52, 2006.

Gillet F., De Foucault B. et Julve Ph. La Phytosociologie synusiale INTÉGRÉE -OBJET ET CONCEPTS. CANDOLLEA 46 : 315-340, 1991.

Glenn H. Aperçu général au MDP et le marché Carbone, Programme UNEP, RISOE, Projet CD4CDM, Atelier sectoriel énergie, Alger, 21 p, 2008.

Guillaumet J.-L. \& Adjanohoun E. La végétation de la Côte d'Ivoire. Le milieu naturel de la Côte d'Ivoire. Mémoires de l’ORSTOM 50 :157-263, 1971.

Kahn F. La reconstitution de la forêt tropicale après une culture traditionnelle (Sud-Ouest de la Côte d'Ivoire). Mémoires de l'ORSTOM 97:1-150, 1982.

Kassi N.J. Successions secondaires post-culturales en forêt dense semidécidue (Côte d'Ivoire): nature, structure et organisation fonctionnelle de la végétation. Thèse de Doctorat, Université de Picardie Jules Verne d'Amiens (France), 212 p, 2006.

Koné M., Kouadio Y.L., Neuba D. F.R., Malan D. \& Coulibaly L. Evolution de la couverture forestière de la Côte d'Ivoire des années 1960 au début du $21^{\mathrm{e}}$ siècle. International Journal of Innovation and Applied Studies 7 (2): 782-794, 2014.

Minang P. A., Jungcurt S., Meadu V., Murphy D. Négociations de la REDD. L'avancée vers Copenhague. 2009. International Institute for Sustainable Development (IISD), ASB Partnership for the Tropical Forest Margins, Manitoba Canada, 161 p, 2009.

Nolte C., Kotto-Same J., Moukam A., Thenkabail P.S., Weise S.F. \& Zapfack L. Land use characterization and estimation of carbon stock in the alternative to slash and burn Benchmark area in Cameroon. Resource and crops management research monograph 28. IITA, Ibadan. 83 p, 2001. 
Peltier R., Njiti F.C., Ntoupka M., Manlay R., Henry M., Morillon V. Evaluation du stock de carbone et de la productivité en bois d'un parc à karités du Nord-Cameroun. Revue Bois et Forêts des Tropiques 294 (4): 3950, 2007.

Saïdou A., BalogounI., Koné B., Gnanglè C., Aho N. Effet d'un système agroforestier à karité (Vitellaria paradoxa c.f. gaertn) sur le sol et le potentiel de production du maïs (Zea maize) en zone soudanienne du Bénin. Int. J. Biol. Chem. Sci. 6 (5): 2066-2082, 2012.

Tayo G.Y.K. Dynamique de la biodiversité ligneuse et des stocks de carbone dans les systèmes agroforestiers à base de cacaoyer au centre Cameroun : cas de Ngomedzap (Cameroun). Mémoire de Master, Faculté Agronomique et des Sciences agricole Université de Dschang (Cameroun), 91 p, 2014.

Torquebiau E.F., Les associations agroforestières et leurs multiples enjeux. Bois et Forêts des Tropiques 271 : 23-34, 2002.

Traoré K., Ganry F., Oliver R., Gigou J. Litter production and soil fertility in a Vitellaria paradoxa parkland in a catena in southern Mali. Arid Land Res. Manag.18(4) : 359-368, 2004. 